\title{
User Engagement with Generous Interfaces for Digital Cultural Heritage
}

\author{
Robert Speakman ${ }^{10000-0001-5578-703 X]}$, Mark M Hall ${ }^{20000-0003-0081-4277]}$, \\ and David Walsh ${ }^{1}[0000-0003-2972-8233]$ \\ 1 Edge Hill University, Ormskirk, Lancashire, UK \\ 2 Martin-Luther-Universität Halle-Wittenberg, Von-Seckendorff-Platz 1, 06120 Halle, \\ Germany robert.speakman@edgehill.ac.uk \\ 3 mark.hall@informatik. uni-halle.de \\ 4 david.walsh@edgehill.ac.uk
}

\begin{abstract}
Digitisation has created vast digital cultural heritage collections and has spawned interest in novel interfaces that go beyond the search box and aim to engage users better. In this study we investigate this proposed link between generous interfaces and user engagement. The results indicate that while generous interfaces tend to focus on novel interface components and increasing visit duration, neither of these significantly influence user engagement.
\end{abstract}

Keywords: Digital Cultural Heritage - Generous Interfaces · User Engagement.

\section{Introduction}

Cultural Heritage institutions have embraced efforts to digitise their extensive collections, making them available to the general public and opening up our cultural heritage. These efforts have created large digital collections [12], but access remains through the search box. However, there has been interest in more "generous" interfaces that go beyond simply providing "1-10 of 10000" results.

These "generous" interfaces are primarily aimed at non-expert users who often find the search box and the need to formulate and interpret queries a major obstacle $[17,16]$. As a result digital cultural heritage (DCH) websites often have very high bounce rates with up to $60 \%$ of users leaving in the first ten seconds. The argument for the new interfaces is that by generously offering up the available content, the novice user will engage more with the content and the interface. Unlike traditional search interfaces, which are quite heavily standardised, generous interfaces demonstrate a large amount of variation and it is unclear how this variation affects their ability to engage users.

In the study presented here we investigate user engagement with three different generous interfaces and attempt to determine how different user interface elements affect user engagement. The remainder of this paper is structured as follows: section 2 discusses the current state of generous interfaces and user engagement, section 3 presents the experiment, section 4 discusses the results, and section 5 concludes with recommendations for future research. 


\section{Generous Interfaces and User Engagement}

To support the novice user in accessing large collections, alternative interfaces have been developed that focus primarily on browsing and visualisation to create richer user experiences [2] that are preferred by non-expert users $[7,15]$. In the field of DCH the labels "generous interfaces" [16] and "rich prospect browsing" [13] have been attached to this type of interface. While both terms are relatively new, they trace their core ideas to the concept of "overview first, zoom and filter, then details on demand" developed in the 90s [14].

In general these kinds of interface initially provide the user with a sample of the content available in the collection or an overview visualisation that highlights the available types of content. This enables the novice user to learn about the collection as a whole [6] and then through browsing and visualisation explore the collection in order to gradually build up a more detailed understanding of the content $[5,8]$.

While user testing has shown that generous interfaces support users in their interaction with DCH collections [3], it is unclear whether they actually manage to engage users in the way they claim. User engagement takes into account both the usability of the interface, but also the users' sense of captivation with the task and system [10,1] and depth of interaction [11]. A number of metrics have been defined for measuring user engagement, but in the study presented here the User Engagement Scale is used in its short-form (UES-SF) [9].

\section{$3 \quad$ Experiment}

\subsection{Methodology}

To investigate user engagement with generous interfaces we developed an on-line experiment and tested three different generous user interfaces. The experiment used a standard interactive information retrieval setup, initially acquiring demographics data, then letting each participant use one of the three interfaces, and finally assessing their experience and engagement.

The first step acquired information on participants' age, gender, education, employment status, and cultural heritage experience. In the second step participants were randomly assigned one of the three tested interfaces. To test the generous interfaces' open-ended exploration support we used the open-ended task instructions from [4], which instructed participants to freely explore until they had enough. The experiment automatically tracked the time participants spent in the system. While showing participants more than one interface was considered, this would have increased the experiment duration and from our experience in on-line settings this would lead to high drop-out rates.

After completing step two, participants were asked to rate how much use they made of the available user interface components. Then participants were asked to assess whether they had used similar interfaces before, whether the initial set of items they saw was interesting, and whether they looked at items that they would not normally be interested in. Finally the UES-SF was administered. 
The experiment was piloted with ten participants and after correcting issues it was made available on-line over a two-week period in November 2017.

\subsection{Tested Generous Interfaces}

As generous interfaces come in a wide range of styles, the decision was made to test three existing, live, production-quality systems, enabling the study to assess engagement in a realistic context. The three interfaces (see Figure 1) were: Discover the Queenslander ${ }^{5}$ (DtQ) [16], the Rijksmuseum's Rijksstudio ${ }^{6}$ (RS), and the Tyne and Wear museum's Collections Dive ${ }^{7}$ (CD) [3].

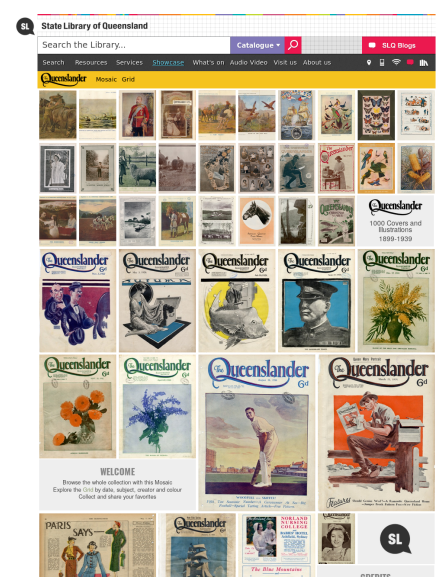

a)

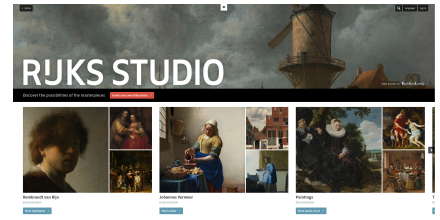

b)

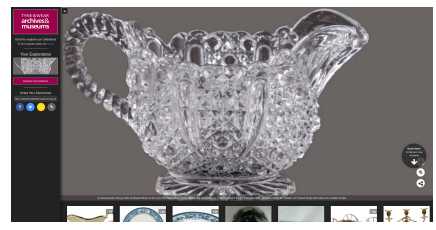

c)

Fig. 1. Screenshots showing the initial view presented by each of the three tested interfaces: a) Discover the Queenslander, b) Rijksstudio, c) Collections Dive.

The DtQ system contains a collection of illustrations from the Queenslander newspaper between the years 1866 and 1939 [16]. It uses an image grid to give the user a generous overview over the data and help them explore it. Additionally it supports exploration via colour and a time-line. It is representative of generous interfaces that aim to surface a very specific collection by providing a visualisation and browsing-based interface.

The RS system lets users curate their own galleries out of the Rijksmuseum's digital collections, which can then be explored via browsing. Unlike the other systems, it includes a curated aspect with galleries created by the Rijksmuseum's curators. Like the DtQ it also supports exploration via colour. The RS interface is heavily influenced by social sharing sites (Tumblr, Pinterest, ...). It is included as an example of a mixed expert-driven and crowd-sourced generous interface.

\footnotetext{
${ }^{5}$ http://www.slq.qld.gov.au/showcase/discover-the-queenslander

${ }^{6}$ https://www.rijksmuseum.nl/en/rijksstudio

7 http://www.collectionsdivetwmuseums.org.uk/
} 
The CD interface is the most novel interface in that it allows exploration of the collection simply by scrolling down the page. It initially shows a randomly selected set of related items and when the user scrolls down, the system either shows more similar items (slow scrolling) or switches to showing very different items (fast scrolling). It is representative of generous interfaces that attempt to provide a very different interaction pattern and user experience.

\subsection{Participants}

Participants were recruited via social media (Facebook, Twitter, LinkedIn) and via physical and electronic noticeboards at Edge Hill University. A total of 620 participants were recruited of which 56 completed the experiment (9\%). Most drop-outs occurred on the initial landing page, a common scenario when inviting participants via social media.

Of the 56 participants 32 were male, 23 female, and 1 undisclosed. While the largest group is from the student age-bracket 18-25 (24), there is a good distribution across the other age brackets as well 26-36 (9), 36-45 (12), 46-55 (7), 56-65 (3), over 65 (1). 24 participants identified as students, 27 as in employment, and the remaining 5 were undeclared. All participants undertook the experiment on-line, using their own devices in their own environment.

\section{Results \& Discussion}

Participants were automatically balanced across the three interfaces. To ensure that this did not introduce any accidental biases, we tested for potential biases due to $\mathrm{CH}$ experience and previous exposure to generous interfaces and found no significant influence by either of these aspects.

Table 1. Participants' visit duration, whether they found the initial items interesting, discovered novel content, and their engagement. Values are formatted [mean (standard deviation)] and, excepting the visit duration, are on a scale of 1 (low) to 5 (high).

\begin{tabular}{|l|c|c|c|}
\hline & DtQ & RS & CD \\
\hline Visit duration & $4 \mathrm{~m} 13 \mathrm{~s}(6 \mathrm{~m} 29 \mathrm{~s})$ & $2 \mathrm{~m} 30 \mathrm{~s}(4 \mathrm{~m} 7 \mathrm{~s})$ & $2 \mathrm{~m} 18 \mathrm{~s}(2 \mathrm{~m} 1 \mathrm{~s})$ \\
Item Interest & $2.58(1.26)$ & $3.38(1.2)$ & $3(1.37)$ \\
Novel Items & $3.47(1.35)$ & $3.52(1.47)$ & $3.7(0.96)$ \\
\hline User Engagement & 3.43 & 4.24 & 4.12 \\
\hline
\end{tabular}

Table 1 shows the main results. All three interfaces are successful in introducing participants to the collections and showing them items they had previously not seen (novel items). While the visit duration is higher for the DtQ, the difference is not statistically significant. Likewise, there is no statistically significant difference on the initial item interest either. However, the DtQ is significantly less engaging than either the RS or the CD (Wilcoxon rank-sum $p<0.05$ ). 
An interesting result is how long some participants spent on the DtQ, even though its engagement score is significantly lower. Clearly visit duration is not a predictor for engagement, even though longer visit times are frequently noted as an aim for generous interfaces.

Potentially the engagement difference is due to the type of data in the three collections. Although initial item interest is not significantly different across the three interfaces, for the RS and the CD there is a significant correlation between initial item interest and engagement (RS: $\rho=0.6, p<0.01, \mathrm{CD}: \rho=0.75, p=$ $0.001)$, which does not exist for the DtQ. The exact nature of this effect needs further study.

The CD's use of scrolling to navigate is novel and clearly manages to engage users. At the same time it has the lowest average visit duration and the lowest standard deviation. We believe that this is because, while the interface engages, the lack of control leads to a relatively consistent point in time where the user has had enough, and since they cannot focus their exploration, they leave.

The generous interface literature focuses on the impact of interface components on engagement, but, with two exceptions, we find no significant correlation between component use and user engagement. For the CD use of scrolling weakly correlates with engagement $(\rho=0.53, p=0.04)$, as does use of the image viewing component in the $\operatorname{DtQ}(\rho=0.47, p=0.04)$.

\section{Conclusion}

Generous interfaces have been put forward as a solution to the high bounce rates experienced by DCH sites. In this paper we presented a study of user engagement with three such interfaces. The central result is that while work on generous interfaces tends to focus on increasing visit durations and developing novel interfaces and visualisations, the results of our study show little influence of these aspects on user engagement. However, what items a user initially sees does significantly correlate with engagement for two of the interfaces.

This strongly indicates that the research focus needs to change from time and novel interfaces to actually understanding novice users' needs and information journeys and then developing interfaces that can support them through these, with a particular focus on the initial interaction moments and the data the users see at that point.

The study has some limitations due to its nature. Three live systems were tested, which did not allow us to track participants' interactions with the interfaces, instead we relied on participants' self-assessment regarding which aspects of the interfaces they used. Additionally the relatively small sample sizes of between 16 and 21 participants per interface limits the strength of our conclusions.

\section{References}

1. Attfield, S., Kazai, G., Lalmas, M.: Towards a science of user engagement (Position Paper). WSDM Workshop on User Modelling for Web Applications (2011), http://www.dcs.gla.ac.uk/ mounia/Papers/engagement.pdf 
2. Bates, M.J.: What is browsing-really? a model drawing from behavioural science research (4) (2007)

3. Coburn, J.: I dont know what Im looking for: Better understanding public usage and behaviours with Tyne \& Wear Archives \& Museums online collections. In: MW2016: Museums and the Web 2016 (2016), http://mw2016.museumsandtheweb.com/paper/i-dont-know-what-im-lookingfor-better-understanding-public-usage-and-behaviours-with-tyne-wear-archivesmuseums-online-collections/

4. Gäde, M., Hall, M., Huurdeman, H., Kamps, J., Koolen, M., Skov, M., Toms, E.G., Walsh, D.: Overview of the SBS 2015 Interactive Track (2015)

5. Giacometti, A.: The Texttiles Browser: An Experiment in Rich-Prospect Browsing for Text Collections. Ph.D. thesis, Faculty of Arts (2009)

6. Hibberd, G.: Metaphors for discovery : how interfaces shape our relationship with library collections. The Search Is Over! Exploring Cultural Collections with Visualization International Workshop in conjunction with DL2014 (September 2014) (2014)

7. Lopatovska, I., Bierlein, I., Lember, H., Meyer, E.: Exploring requirements for online art collections. Proceedings of the Association for Information Science and Technology 50(1), 1-4 (2013)

8. Mauri, M., Pini, A., Ciminieri, D., Ciuccarelli, P.: Weaving data, slicing views: A design approach to creating visual access for digital archival collections. ACM International Conference Proceeding Series CHItaly pp. 1-8 (2013). https://doi.org/10.1145/2499149.2499159

9. OBrien, H.L., Cairns, P., Hall, M.: A practical approach to measuring user engagement with the refined user engagement scale (ues) and new ues short form. International Journal of Human-Computer Studies 112, 28 - 39 (2018). https://doi.org/https://doi.org/10.1016/j.ijhcs.2018.01.004, http://www.sciencedirect.com/science/article/pii/S1071581918300041

10. Peters, C., Castellano, G., de Freitas, S.: An exploration of user engagement in HCI. Proceedings of the International Workshop on Affective-Aware Virtual Agents and Social Robots pp. 9:1-9:3 (2009). https://doi.org/10.1145/1655260.1655269, http://doi.acm.org/10.1145/1655260.1655269

11. Peterson, E.T., Carrabis, J.: Measuring the Immeasurable: Visitor Engagement p. 54 (2008)

12. Petras, V., Hill, T., Stiller, J., Gäde, M.: Europeana - asearch engine for digitised cultural heritage material. Datenbank-Spektrum 17(1), 41-46 (Mar 2017). https://doi.org/10.1007/s13222-016-0238-1, https://doi.org/10.1007/s13222-0160238-1

13. Ruecker, S., Radzikowska, M., Sinclair, S.: Visual Interface Design for Digital Cultural Heritage: A Guide to Rich-Prospect Browsing (Digital Research in the Arts and Humanities). Routledge (2016)

14. Shneiderman, B.: The eyes have it: a task by data type taxonomy for informatio nvisualizations. Proceedings 1996 IEEE Symposium on Visual Languages pp. 336343 (1996). https://doi.org/10.1109/VL.1996.545307

15. Walsh, D., Hall, M.M., Clough, P., Foster, J.: The ghost in the museum website: Investigating the general public's interactions with museum websites. In: TPDL'17 (2017)

16. Whitelaw, M.: Generous Interfaces for Digial Cultural Collections. DHQ: Digital Humanities Quarterly 9(1) (2015)

17. Wilson, M.L., Elsweiler, D.: Casual-leisure searching: the exploratory search scenarios that break our current models. In: Proceedings of HCIR. pp. 28-31 (2010) 